\title{
La Voce dei Pazienti Dare la vita o dare problemi? La domanda da un milione di dollari
}

\author{
Ma anche le risposte qui hanno grande valore. \\ Una carrellata di esperienze, brevi biografie, riflessioni e pareri su una delle \\ decisioni più cruciali per chi è affetto da una malattia ereditaria. \\ Niente consigli, ma molti inviti alla consapevolezza.
}

L.P.: Ciao a tutti e a tutte! Ho ereditato da parte di padre il rene policistico. Mi sono controllata sempre e fino a due anni fa non avevo nulla. La settimana scorsa eco di controllo e... sorpresa! Sono apparse le maledette cisti. I miei reni funzionano benissimo, la mia pressione è okay. Insomma diciamo che per ora, a parte le cisti, sto benissimo. Arriva la domanda da un milione di dollari. Ho 30 anni e vorrei avere un figlio. Sono consapevole che potrei trasmettere il gene della malattia a mio figlio. Cosa mi consigliate? Esami/visite/controlli... ogni consiglio, esperienza, informazione è tutto gradito. GRAZIE!

A.M.: Sono ora al termine della mia gravidanza. Devo dire che fin dall'inizio non mi hanno fatto vivere questa gravidanza in modo sereno, perché ti mettono davanti a tutti i rischi possibili. Ma questo è il lavoro dei medici. A me è andato tutto bene. Il rischio più grande era che il feto avesse un'insufficienza renale e quindi ci fosse bisogno di un trapianto da subito. Io ero già d'accordo coi medici che in tal caso, dopo l'amniocentesi, avrei proceduto con l'aborto (scelta assolutamente personale). Ma visto che tu, come me, hai quello dell'adulto, problemi non ce ne dovrebbero essere. Punto primo, perché se ce l'avesse comparirebbe in età adulta, punto secondo, perché hai $50 \%$ di probabilità di trasmetterglielo. Detto questo, sii solo consapevole di quello che POTREBBE essere, ma non farti problemi a cercare un figlio e a volte tappati le orecchie e vai avanti, se ci credi davvero. Trova una brava ginecologa, un genetista e un nefrologo che ti seguano e vedrai che tutto andrà bene! Incrocio le dita per te!

F.G.: Io ho due bellissimi figli, di 24 e 17 anni, tutti e due policistici. Hanno ereditato la malattia da mio marito che l'ha ereditata da sua madre. Non mi sento di dare consigli, è una scelta molto personale. Ti dico solo che non potrei pensare la mia vita senza di loro, ma vedendo i loro problemi che iniziano a manifestarsi mi sento in colpa. Cosa avrei potuto fare? Solo decidere di non averli. All'epoca del primo, mi disse- ro che la trasmissione della malattia era molto improbabile. L'ho controllato sempre e fino a 5 anni non c'era niente, credevo di averla scampata. Poi, il secondo figlio, in occasione dell'ecografia alle anche che fanno poco dopo la nascita, decisi di ricontrollare anche il grande, che aveva 7 anni e il medico mi disse: "Sì, sì signora, ci sono le cisti, è bene portarlo a Pisa, che sono più esperti". Da li è iniziato tutto il nostro percorso. Due anni fa ho fatto controllare anche il secondo e ho avuto la conferma che anche lui è malato. Che dire? A volte vorrei chiedere a loro se abbiamo sbagliato, ma credo che la vita sia un dono meraviglioso sempre, perciò ascolta il tuo cuore e decidi quello che credi sia meglio per voi. Un abbraccio.

A.B.: Ciao, io partecipai a un convegno dell'AIRP dove un nefrologo parlava della possibilità di avere figli senza trasmettere la patologia, attraverso la modifica del gene difettoso. Disse che non è una pratica che fanno qui in Italia $\mathrm{e}$ che è costosa. Disse pure che ci si poteva rivolgere a lui per informazioni, altro non ascoltai perché cosa può interessare a una 19 enne di bambini...?

R.N.: Ciao, io ho una cisti sul rene sx e una sul dx, poi microcisti. All'ultimo controllo ecografico di martedì mi hanno detto che non posso essere definita policistica! Sorpresa, da 15 anni mi ritenevo tale! Fatta sempre prevenzione, vita sana, pressione sempre monitorata, sport, dieta iposodica ecc..., ho una bimba stupenda di tre anni e mezzo, il nefrologo che mi segue mi ha detto di non fare nulla fino ad almeno i 10-12 anni. Ha sempre sostenuto la gravidanza senza creare allarmismi inutili. Vai tranquilla!!! In bocca al lupo!!!

A.I.: Io ho due bimbi, il secondo dei quali portatore di reni policistici. I problemi li ho avuti nella prima gravidanza, perché essendo ipertesa, la pressione è salita troppo è ho dovuto partorire a 35 settimane. La seconda gravidanza è andata benissimo. Auguri! 
L.S.: Ciao io ho due figli di 19 e 22 anni. Io ho i reni policistici ma - incrociamo le dita - loro sono sani.

C.B.: Cara L., ti consiglio di leggere il libro dedicato ai pazienti e ai loro familiari che puoi richiedere sul sito dell'AIRP perché dà molte spiegazioni, è fatto molto bene. Il titolo è: Rene Policistico Autosomico Dominante. Parlarne è già combatterlo. Se hai deciso di avere bimbi, sappi che devi avere grande forza e coraggio, perché può salire la pressione arteriosa... Ciao e buon proseguimento!

M.R.: Mio marito è ammalato. Abbiamo tre figli. Il secondo comincia adesso, a 15 anni, ad avere cisti. Gli altri due sono sani per il momento. Quando abbiamo deciso di averli non sapevamo ancora della malattia di mio marito. Tornassi indietro li rifarei tutti e tre. Spero nella ricerca e che quando ne avranno bisogno ci sarà una cura. Fai tutti i figli che vuoi e che puoi. Saranno la tua gioia e la tua forza. In bocca al lupo!!!

M.S.: Io mi sono accorta di essere policistica per caso, a quasi 50 anni. Prima nessuna diagnosi finché l'ecografia non è entrata nella prassi. Entrambi i reni quasi out con funzionalità $30 \%$. Avevo già due figlie (poi risultate sane) e per ora anche nipoti sani. I miei genitori e mia sorella ok. Pare che invece il problema arrivi dalla famiglia della bisnonna paterna, peraltro morta negli anni '40 ultrasettantenne. Perché vi racconto la mia biografia? Perché la paura della malattia è spesso essa stessa una malattia... invece bisogna pensare positivo.

P.R.: Io ho cisti dalla nascita, ho 32 anni e da quando ne ho 18 prendo la pastiglia per la pressione alta. Adesso sto bene, ammetto di non curare molto l'alimentazione ma... ti direi di fare quello che ti senti. Io ho paura, mia mamma è in dialisi, e quando era incinta ha rischiato il blocco renale, ma come vedi ci sono nel gruppo persone che hanno avuto più figli (io sono figlia unica), stanno bene e non hanno avuto problemi. C'è chi è andato in dialisi giovane pur non avendo avuto figli quindi fai quello che senti, solo fatti seguire e consigliare!

P.O.: Non serve nessun controllo... c'è la possibilità del $50 \%$. Ma non è un "testa o croce", è un figlio/a. Se lo desideri cosi tanto è un tuo diritto di donna, un tuo sogno, il tuo futuro... allora fallo!!! Sii soltanto consapevole del fatto che potrebbe (speriamo di NO mille volte!!!) avere il rene policistico. In quel caso, non condanni tanto te stessa a una vita un po' più difficile, ma lui o lei. Cioè, quel che conta è la consapevolezza. Avere un figlio è la cosa più meravigliosa a questo mondo... dare la vita a un nuovo essere umano è il gesto più nobile che esista... ma è anche un fatto egoistico... scegli tu per lui/lei. Io ho un fratello. Lui ha scelto di sì. Ha una bimba bellissima di 6 anni che adora e che per sua scelta non le ha mai neppure misurato la pressione. E la gioia dei nostri genitori e mia, oltre che sua e di sua moglie. Mio fratello è in dialisi e avrà fatto in 42 anni di vita solo una settimana di ricovero ospedaliero.

Io 40 anni, di cui quindici passati entrando e uscendo dall'Ospedale, ora trapiantato ma con alle spalle due nefrectomie (asportazione dei due reni), altri tre interventi e più di tre anni di dialisi... e ho scelto NO. Quindi tutto dipende dal proprio vissuto. Se hai paura ... non lo fare, se lo/la desideri tantissimo... FALLO, non pensare alle conseguenze e vivi serenamente. Insomma, non esiste una scelta sbagliata o giusta, esiste solo la tua scelta e l'amore che nel caso riuscirai a trasmettere a tuo figlio.

M.P.: Ciao L. Io ho scoperto il rene policistico solo sei anni fa, dopo il secondo figlio. Nessuno della mia famiglia fino ai bisnonni soffriva di rene policistico. Perciò, faccio i controlli ai miei bimbi: il grande di 7 anni ha una cisti renale e il piccolo di 5 un rene più piccolo. È vero, la medicina va avanti, ma io vivo con angoscia i controlli dei miei bimbi e anche se sono la mia vita non so se avrei fatto figli, anche se sicuramente avrei adorato i bambini. Ora prego solo che i miei figli non sviluppino la malattia.

S.V.: Io a 24 anni ero "sana". A 25 decido di avere un figlio, gravidanza tranquilla fino al $7^{\circ}$ mese, poi gestosi e quindi cesareo urgente... Nasce mio figlio e scopro che non solo io ho ereditato la malattia ma l'ho anche trasmessa a mio figlio. Che dire? Io oggi parlo da trapiantata, ho 43 anni e un figlio di 17 che gode di ottima salute. I figli sono la cosa più bella del mondo e non rimpiango di averlo messo al mondo, però se avessi saputo prima di essere policistica ci avrei pensato un attimo in più! Adesso non ci resta che sperare nella ricerca!

O.S.: Ho scoperto di essere policistica a 37 anni, dopo due gravidanze. I miei figli (ora di 38 e 34 anni) sono sani e dall'analisi del DNA risulta che non sono policistici. Lo so, è andata bene, ma credo che la scelta di avere figli, anche rischiando che siano malati, sia assolutamente personale. Ricorda poi che la ricerca fa passi in avanti ogni giorno anche grazie ad AIRP!!

L.P.: Il rischio c'è... come ce l'avevano anche se inconsapevolmente i miei genitori... però hanno messo al mondo tre figli! Siamo tutti e tre sani, belli e con una vita meravigliosa! A 30 anni sono comparse le cisti è vero! Però sono grata ogni giorno a chi mi ha messo al mondo. 